\title{
PAP SMEAR FOR SCREENING T. VAGINALIS
}

Sateesh. K. Malkappa, Saileela. K, Rajendra. B. Surpam, Trinain Kumar Chakraverti

\author{
1. Assistant Professor, Department of Microbiology, Kamineni Institute of Medical Sciences. Sreepuram, \\ Narketpally. Dist. Nalgonda. Andhra Pradesh. India. \\ 2. Professor \& HOD, Department of Microbiology, Kamineni Institute of Medical Sciences. Sreepuram, \\ Narketpally. Dist. Nalgonda. Andhra Pradesh. India. \\ 3. Professor, Department of Microbiology, Kamineni Institute of Medical Sciences. Sreepuram, Narketpally. \\ Dist. Nalgonda. Andhra Pradesh. India. \\ 4. Assistant Professor, Department of Microbiology, Kamineni Institute of Medical Sciences. Sreepuram, \\ Narketpally. Dist. Nalgonda. Andhra Pradesh. India.
}

\section{CORRESPONDING AUTHOR}

Dr. Sateesh K Malkappa. Asst Prof.

Department of Microbiology. Kamineni Institute of Medical Sciences.

Sreepuram, Narketpally. Dist. Nalgonda.

Andhra Pradesh. India. 508254

E-mail: drsateesh2006@gmail.com,

Ph: 00919885962543

ABSTRACT: BACKGROUND: Trichomonas vaginalis is sometimes seen in Papanicolaou stained smears, but because emphasis is placed on malignant cells in Papanicolaou stained smears, not much is done to search for this parasite in smears. In this study, cervical and vaginal specimens were examined by conventional Papanicolaou method for the presence of Trichomonas vaginalis microscopically. MATERIALS AND METHODS: Five hundred high vaginal swabs collected from gynaecology OPD were stained with Papanicolaou stain. RESULTS: One hundred and fifty (30\%) out of 500 Papanicolaou stained smears screened, were positive for Trichomonas vaginalis. Out of them $76.67 \%$ of positive smears were from asymptomatic females. Presumptive diagnosis based on perinuclear halo and complete T. vaginalis had the highest sensitivity of $69.33 \%$, while diagnosis based on perinuclear halo alone was $50.66 \%$ and 41.33\% for diagnosis based on identification of complete organisms in Pap smear.

KEY-WORDS: Papanicolaou smears, Trichomonas vaginalis, perinuclear halo,

INTRODUCTION: Trichomonas vaginalis is a protozoan pathogen of the human urogenital tract. The prevalence of Trichomonas vaginalis has been reported to be as high as $26 \%$ among female STD clinic patients and $22 \%$ among HIV-positive women. ${ }^{1}$ In infected female patients, symptoms include: vaginal discharge, vulvar pruritis, dysuria, and dyspareunia. Classical green, frothy, foul-smelling discharge occurs in $10 \%$ of the women. However, up to $50 \%$ of the infected female patients are asymptomatic. ${ }^{2}$ In women, trichomoniasis may play a role in development of cervical neoplasia, postoperative infections, and adverse pregnancy outcomes and as a factor in atypical pelvic inflammatory disease and infertility. ${ }^{3}$ The presence of $\mathrm{T}$. Vaginalis in the vagina increases predisposition to HIV seroconversion. Having trichomoniasis may increase the chance that an HIV infected woman passes HIV to her sex partner(s). ${ }^{4,5}$

Diagnosis of $\mathrm{T}$. vaginalis is an important public health issue, as asymptomatic patients may act as a reservoir for its transmission to their sexual partners. Wet mount preparations had a sensitivity of 40 to $75 \%$ while T. vaginalis culture had a sensitivity of 86 to $97 \% .6,7$ However, expert personnel and laboratory support are required and they are not readily available in a primary care setting. ${ }^{2}$ For direct microscopy only fresh specimens are of value. ${ }^{8}$ Diagnosis by 
wet-mount requires visualisation of viable, motile protozoa; therefore, specimens must be examined immediately. The sensitivity of wet-mount microscopy can be further reduced as a result of delays between specimen collection and examination, ${ }^{7}$ and with the large numbers of patients attending gynaecological clinics, an 'on the spot' examination of a vaginal swab is virtually impossible. ${ }^{9}$

Stained smears have the advantage that there can be considerable delay between preparation and staining and examination of a smear without loss of reliability in diagnosis. In primary care setting, the Papanicolaou (PAP) smear is a commonly performed screening test for cervical cancer. Thus, detection of $\mathrm{T}$. vaginalis in PAP smear would mean an additional advantage provided the result is accurate. ${ }^{2}$ Trichomonas vaginalis is sometimes seen in PAP smears where it is reported, but because emphasis is placed on malignant cells in PAP smears, not much is done to search for this parasite in smears. ${ }^{3}$ Moreover, studies have shown that PAP smear may detect Trichomonas vaginalis in a considerable number of culture-negative women. ${ }^{10}$ The aim of this study was to determine the suitability of PAP smear in the detection of Trichomonas vaginalis in cervical and vaginal specimens. ${ }^{2}$

MATERIALS AND METHODS: A total of 500 high vaginal swabs were collected from females in reproductive age group attending at gynaecology OPD. These smears were stained by conventional Papanicolaou method, ${ }^{11}$ and screened microscopically for Trichomonas vaginalis. The study was carried in department of microbiology, during the period of August 2010 August 2011.

PAPANICOLAOU METHOD ${ }^{11}$ : Each specimen was smeared on a clean grease free slide and fixed in ether-alcohol for 30 minutes. The specimens were then stained by the Papanicolaou method as follows: Harris's haematoxylin without acetic acid for 5 minutes, rinsed in tap water and differentiated in 1\% acid alcohol for 30 seconds and blued in Scott's water for 2 minutes. Smears were taken to $95 \%$ alcohol and stained in OG6 for 2 minutes, rinsed in $95 \%$ alcohol and stained in EA 35 for 2 minutes. Smears were then taken to two changes of absolute alcohol, xylene and mounted in DPX. The stained smears were examined under the light microscope at low and high power objectives for the presence of Trichomonas vaginalis and perinuclear halo.

Identification of one or more of the following morphological characters was considered to be conclusive of T. vaginalis: a pear-shaped, oval to round, cyanophilic organism that ranges in size from 15-30 microns; pale nucleus, vesicular and centrally located; cytoplasmic eosinophilic granules or flagella. The presence of perinuclear halo in the epithelial cells was also used as a presumptive diagnosis for T. vaginalis. These criteria were also used to distinguish trichomonads from cytoplasmic fragments.

RESULTS: One hundred and fifty (30\%) out of 500 PAP smears screened were positive for Trichomonas vaginalis. On retrospective analysis $76.67 \%$ of positive smears were from asymptomatic females. Only $23.33 \%$ had history of vaginal discharge and pruritis. $25(5 \%)$ of 150 positive PAP smears showed Candida infection and all were symptomatic women. See table1

Presumptive diagnosis based on perinuclear halo alone was $50.66 \%$ while diagnosis based on identification of organisms alone in PAP smear was $41.33 \%$. When both the morphological characters of Trichomonas vaginalis were given equal consideration for 
identification, i.e slide was considered positive when any one or both the characters are present; diagnosis of Trichomonas vaginalis rose to the sensitivity of $100 \%$. See table 2 .

DISCUSSION: Papanicolaou is the best staining method in cytology, because it helps to effectively differentiate malignant cells from non-malignant cells. It also stains the cytoplasm and its contents. Its ability to differentiate acidophilic materials from basophilic materials as well as its ability to stain non-cellular substances such as fibrin, crystals and pigments, makes it an essential stain in. T. vaginalis, the causative organism for trichomoniasis is the most common curable sexually transmitted organism worldwide. It parasitizes both males and females where it is sometimes asymptomatic in the early stages of the infection. T. vaginalis infection is said to play a role in the development of cervical neoplasia, postoperative infections, and adverse pregnancy outcomes and as a factor in atypical pelvic inflammatory disease and infertility. In our study $76.67 \%$ of females in reproductive age group were asymptomatic carriers of $\mathrm{T}$. Vaginalis, and are at risk of developing above said complications until treated in time. There is also epidemiological and experimental evidence that PAP smears are beneficial in detecting infections that are risk factors associated with cervical cancer. It can detect certain viral, bacterial, and fungal infections of the cervix and vagina. Culture is a very sensitive method of detecting $\mathrm{T}$. vaginalis but it is expensive and time consuming.

In the present study very broad criteria were used for the identification of Trichomonas vaginalis in PAP smears, to increase the sensitivity. Twenty three percent of the women with Trichomonas - positive PAP smears had genital symptoms, while $77 \%$ of women were asymptomatic carriers of Trichomonas vaginalis. Thus our data suggest that PAP smear may detect Trichomonas vaginalis in a considerable number of asymptomatic women. Steven KF

Loo. et $\mathrm{al}^{2}$ reported that Pap smear could diagnose $\mathrm{T}$. vaginalis infection in $42 \%$ of asymptomatic carriers.

The comparison of positive results showed that the highest sensitivity was found when diagnosis was based on perinuclear halo and/or T. vaginalis in PAP smear followed by sensitivity of $50.66 \%$ when diagnosis was based on perinuclear halo alone. Presumptive diagnosis based on identification of complete organisms alone in Pap smear was $41.33 \%$. Avwioro $\mathrm{O} \mathrm{G}^{3}$ also reported a similar sensitivity pattern of $65.77 \%, 52.63 \%$ and $42.11 \%$ for diagnosis based on both perinuclear halo and T. vaginalis, perinuclear halo alone and complete organisms in PAP smear respectively. In our study, sensitivity for the PAP smear was $100 \%$ which is in comparison with previous studies in the diagnosis of T. vaginalis. The reported sensitivities ranged from 83 to $99 \% .^{12,13}$

CONCLUSION: Papanicolaou smears used for routine screening of cervical cancers can also be used for screening T. vaginalis infection in females. Papanicolaou smears can detect trichomoniasis in asymptomatic patients and is suggested to be one of the best screening tools for asymptomatic carriers of T.vaginalis in females. This helps in early detection and treatment of infection.

\section{REFERENCES:}

1. Frank Sorvillo, Lisa Smith, Peter Kerndt, Lawrence Ash. Trichomonas vaginalis, HIV and African-Americans. Emerging Infectious Diseases 2001;1(6):927-32 
2. Avwioro O G. Diagnosis of trichomoniasis in pap smears; how effective is it. Eur J Exp Bio 2011;1(1):10-13

3. Steven KF Loo, William YM, Tang KK Lo. Clinical significance of Trichomonas vaginalis detected in Papanicolaou smear: a survey in female Social Hygiene Clinic. Hong Kong Med J 2009;15:90-3

4. Cotch MF, Pastorek JG, Nugent RP, Hillier SL, Gibbs RS, Martin DH et al. Trichomonas vaginalis associated with low birth weight and preterm delivery. The Vaginal Infections and Prematurity Study Group. Sex Transm Dis 1997;24:353-60.

5. Moodley P, Connolly C, Sturm AW. Interrelationships among human immunodeficiency virus type 1 infection, bacterial vaginosis, trichomoniasis, and the presence of yeasts. J Infect Dis 2002;185:69-73.

6. Joseph G. Lossick, DO. The diagnosis of vaginal trichomoniasis. J of American Med Assoc 1988;259(8):1230.

7. Thomason JL, Gelbart SM, Sobun JF, Schulien MB, Hamilton PR. Comparison of four methods to detect Trichomonas vaginalis. J Clin Microbiol 1988;26:1869-70.

8. J Sydney and McCann. Comparison of direct microscopy and culture in the diagnosis of trichomoniasis. Brit J vener. Dis 1974;50:450-2.

9. P. R. Mason, Heather Super, and P. J. Fripp. Comparison of four techniques for the routine diagnosis of Trichomonas vaginalis infection J clin Path 1976;29:154-7.

10. C S Petersen, L Carl, D Alnor, U Thomsen, H K Thomsen. Ignored trichomonal infestation diagnosed by Papanicolaou smear. Genitourin Med 1995;71:257-8.

11. Anne M. Specimen preparation. In: John Bancroft, editor. Theory and practice of histological techniques, $5^{\text {th }}$ ed. London: Churchill Livingstone; 2005. p. 87- 105

12. Weinberger MW, Harger JH. Accuracy of the Papanicolaou smears in the diagnosis of asymptomatic infection with Trichomonas vaginalis. Obstet Gynecol 1993;82:425-9.

13. Lara-Torre E, Pinkerton JS. Accuracy of detection of Trichomonas vaginalis organisms on a liquid-based papanicolaou smear. Am J Obstet Gynecol 2003;188:354

Table 1: Prevalence of T.vaginalis in PAP smears

\begin{tabular}{|l|l|l|}
\hline Total number of PAP positive specimens & $\mathbf{1 5 0}$ & $\mathbf{1 0 0} \%$ \\
\hline Presence of genital symptoms & 35 & $23.33 \%$ \\
\hline Absence of genital symptoms & 115 & $76.67 \%$ \\
\hline Concomitant sexually transmitted disease & 25 & $16.66 \%$ \\
\hline
\end{tabular}

Table 2: Comparison of Morphological features for identification of $T$ vaginalis in PAP smears

\begin{tabular}{|l|l|l|}
\hline Investigation & No. & Sensitivity\% \\
\hline $\begin{array}{l}\text { Total positive for perinuclear halo alone (suggestive of T. } \\
\text { vaginalis) }\end{array}$ & 76 & 59.66 \\
\hline Total positive for complete parasite alone seen in PAP smears & 62 & 41.33 \\
\hline Total positive for both perinuclear halo and complete T. vaginalis & 12 & 8 \\
\hline $\begin{array}{l}\text { Total positive for perinuclear halo and complete T. vaginalis: } \\
\text { alone or both }\end{array}$ & 150 & 100 \\
\hline
\end{tabular}

Journal of Evolution of Medical and Dental Sciences/Volume1/Issue5/November-2012Page-840 